\title{
Processive Catalysis
}

\author{
Stijn F. M. van Dongen, * Johannes A. A. W. Elemans, Alan E. Rowan, and \\ Roeland J. M. Nolte*
}

enzymes · host-guest systems .

noncovalent interactions - processivity .

supramolecular chemistry

Nature's enzymes are an ongoing source of inspiration for scientists.
The complex processes behind their selectivity and efficiency is slowly
being unraveled, and these findings have spawned many biomimetic
catalysts. However, nearly all focus on the conversion of small
molecular substrates. Nature itself is replete with inventive catalytic
systems which modify, replicate, or decompose entire polymers, often
in a processive fashion. Such processivity can, for example, enhance
the rate of catalysis by clamping to the polymer substrate, which
imparts a large effective molarity. Reviewed herein are the various
strategies for processivity in nature's arsenal and their properties. An
overview of what has been achieved by chemists aiming to mimic one
of nature's greatest tricks is also included.

\section{Introduction}

Catalysis can be called processive if the catalyst associates with its substrate and then performs multiple rounds of catalysis before dissociation. In rare cases, processivity can express itself through multiple conversions of the same small molecular substrate. In general, however, processive catalysts specialize in polymeric substrates. This specialization is not surprising: with its repeating monomers, the relatively ordered nature of a polymer makes it fertile ground for a catalyst seeking to perform the same reaction over and over again. A distributive catalyst, which follows the conventional association/conversion/dissociation pattern, is at a disadvantage here when compared to a processive one, which only requires a single association to enable a series of conversions.

Such a conveyor belt of potential reaction sites brings the concept of effective molarity into play. In these cases, a catalyst is effectively directed towards its next reaction through its link with the polymer, as it can slide along that thread. ${ }^{[1]}$ Because all its potential reaction sites are also along that thread, the three-dimensional space through which it

[*] Dr. S. F. M. van Dongen, Dr. J. A. A. W. Elemans,

Prof. Dr. A. E. Rowan, Prof. Dr. R. J. M. Nolte

Radboud University Nijmegen, Institute for Molecules and Materials Heyendaalseweg 135, 6525 AJ Nijmegen (The Netherlands)

E-mail: Science@StijnvanDongen.nl

R.Nolte@science.ru.nl would normally diffuse has been reduced to a two-dimensional Dor "onedimensional"? space, virtually increasing the molarity of substrates. With this in mind, it may not come as a surprise that the majority of artificial processive catalysts have a rotaxanelike character. ${ }^{[2]}$ The increase in effective molarity results in remarkably high efficiency and opens the door to sequential catalysis: conversion of substrates according to a predesigned sequence. Given the abundant use of polymers throughout society, and the efficiency of nature in their synthesis and selective modification, it is surprising that the number of biohybrid or fully synthetic processive catalysts developed to date is extremely small. Artificial molecular machines offer great expectations for inroads in this field. ${ }^{[3]}$

The aim of this review is to highlight the modes of actions of natural processive catalysts and to describe initial attempts to mimic their behavior, hopefully stimulating the community to venture further into this area.

\section{Processive Enzymes in Nature}

A fast enzyme needs to easily come into contact with its substrate if it is to leverage its high turnover rate. Similarly, an enzyme with a great substrate affinity could nonetheless be slow in its actual turnovers, thus foregoing the advantage of its great affinity. This balance has been established in enzyme kinetics models such as the Michaelis-Menten model. It states that the efficiency of an enzyme is not only dependent on its maximal catalytic turnover rate $\mathrm{v}_{\max }$, expressed in $\mathrm{s}^{-1}$, but also on its affinity for its substrate, here represented by the Michaelis constant $\mathrm{K}_{\mathrm{m}}$, expressed in $\mathrm{M} . \mathrm{K}_{\mathrm{m}}$ is an inverse measure of binding affinity, so it follows that the most 
efficient enzymes display a combination of a high $\mathrm{v}_{\max }$ with a low $\mathrm{K}_{\mathrm{m}}$. Naturally occurring processive enzymes generally have a remarkable efficiency. These enzymes leverage processivity to manipulate their effective $\mathrm{K}_{\mathrm{m}}$ and achieve greater productivity. The degree of substrate enclosure divides these enzymes in two classes: one where a substrate is completely encircled, and one where a substrate is only partially enclosed. ${ }^{[1]}$

We refer to the cited specialized reviews for alternative forms of natural processivity: noncatalytic motor proteins ${ }^{[4]}$ and nonproteins (ribosomes). ${ }^{[5]}$

\subsection{Protein Rings}

When an enzyme fully encircles a (bio)polymeric substrate, the two are topologically linked, thus forming a rotaxane-like complex. Because of this mechanical interlocking, no highly specific interactions between clamp and template are required. Toroids that encircle nucleic acids, for example, mostly feature a positively charged tunnel and nothing more. A pure example of rotaxane-like processivity is bacteriophage $\lambda$-exonuclease. ${ }^{[6]}$ This toroidal enzyme is a homotrimer (Figure $1 \mathrm{a})$. It degrades DNA in the $5^{\prime}$ to $3^{\prime}$ direction, and explains why only one of the two strands which pass through its cavity is degraded. In addition, the cavity is tapered: one end is large enough to accommodate a double helix, while the other can only fit a single strand.

Opposite in function, certain DNA polymerases also employ toroidal structures. These can involve multiple components. For example, the T4 bacteriophage DNA polymerase gp43 has no inherent processivity. ${ }^{[7]}$ However, it can associate with the homotrimeric toroidal clamp protein gp45. This clamp can almost entirely encircle DNA, thus assuming a $\mathrm{C}$ shape and leaving a single subunit interface opened. It slides over DNA and has no catalytic function, but when the tail of gp43 docks in the clamp's open interface, DNA enclosure is completed and the polymerase is tethered to its template. This docking is dynamic, as additional copies of polymerase can displace previous ones, and is thought to help bypass lesions during DNA replication. ${ }^{[7,8]}$

For recruiting multiple enzymes, it may be easier to envisage a heterotrimer doing the job, which is the case in the archaeon $S$. solfataricus $\mathrm{P} 2 .^{[9]}$ Its clamp-shaped processivity

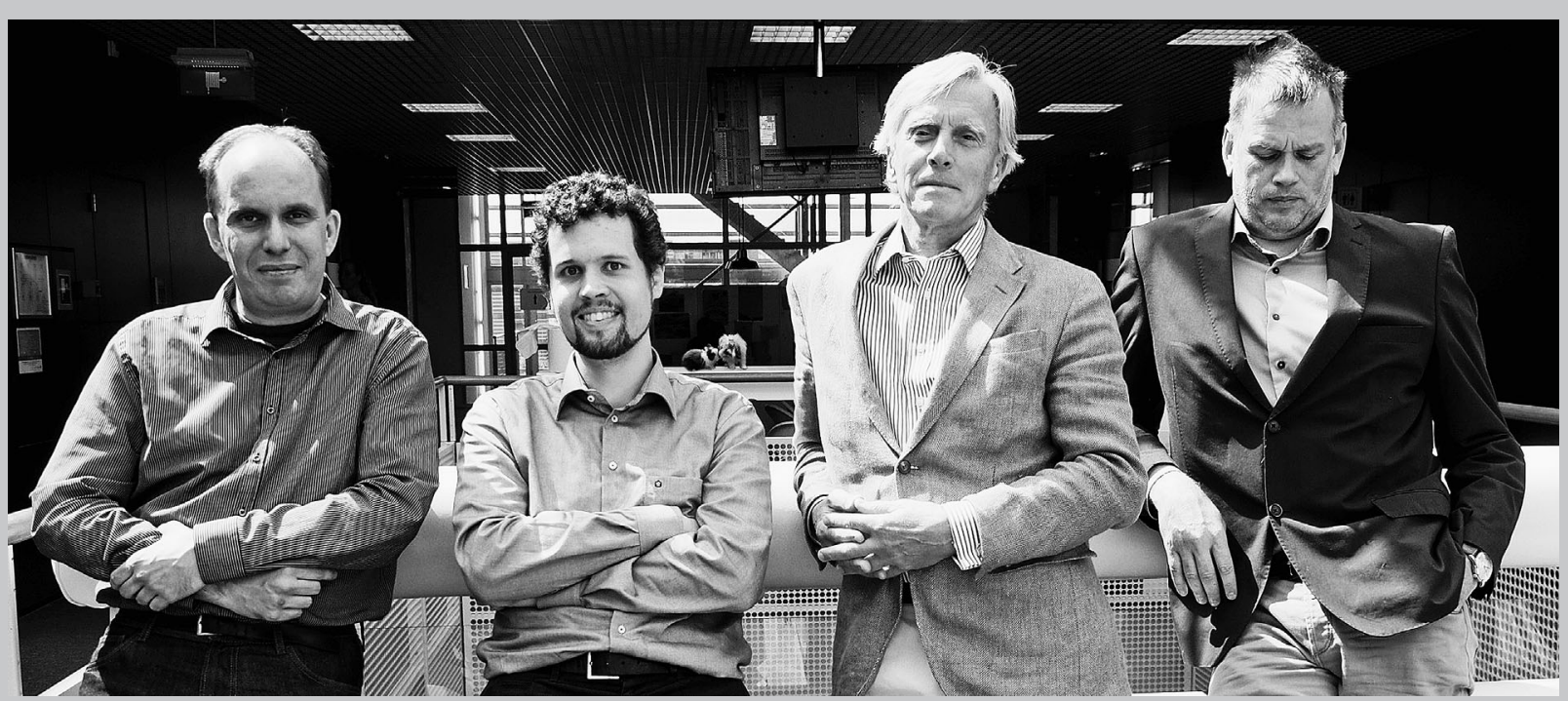

Stijn van Dongen (center left) finished his M.Sc. studies at the ETH Zürich (cum laude) and obtained his Ph.D. in 2010 with Profs. R. J. M. Nolte and J. C. M. van Hest at the Radboud University Nijmegen, working on self-assembled polymersome nanoparticles for catalysis or chemical biology. He then moved to the École Normale Supérieure and the Institut Curie in Paris. With a personal grant, he developed dynamic surface coatings for cell culture with Dr. C. Tribet and Dr. M. Piel. In 2012, he returned to Nijmegen to explore processive protein-based rotaxanes.

Hans Elemans (left) completed his PhD in 2001 with Prof. R. J. M. Nolte at the Radboud University Nijmegen. After that he initiated a cooperation between the Physics and Chemistry departments there, with the aim to study self-assembly and catalytic processes at the single-molecule level using Scanning Probe Microscopy. In 2008 he was in the group of Prof. Steven De Feyter at K. U. Leuven, after which he returned to Nijmegen to start his independent research group. In 2010 he was awarded an ERC Starting Grant.

Professor Dr. Alan Rowan (right) studied at the University of Liverpool, UK, where he obtained a Ph.D. in Physical Organic Chemistry. In 1992 he moved to New Zealand for Postdoctoral study with Prof. C. Hunter. In 1994 he moved to Nijmegen as a Mare Curie Fellow with Professor Prof. R. J. M. Nolte in the area of hierarchal materials. There he became full professor in 2005. He was awarded numerous prizes the most recent being the RSC 2014 Soft Matter and Biophysical Award.

Roeland J. M. Nolte (center right) is a Professor of Organic Chemistry at the Radboud University Nijmegen. He is a member of the Royal Netherlands Academy of Science. His interests span many topics at the interfaces of supramolecular and biomimetic chemistry. His contributions to science were recognized with many awards and prizes including the first Royal Netherlands Academy of Science chair in chemistry, and a knighthood. He served on the editorial boards of many scientific journals, including Science and Chemical Communications. 

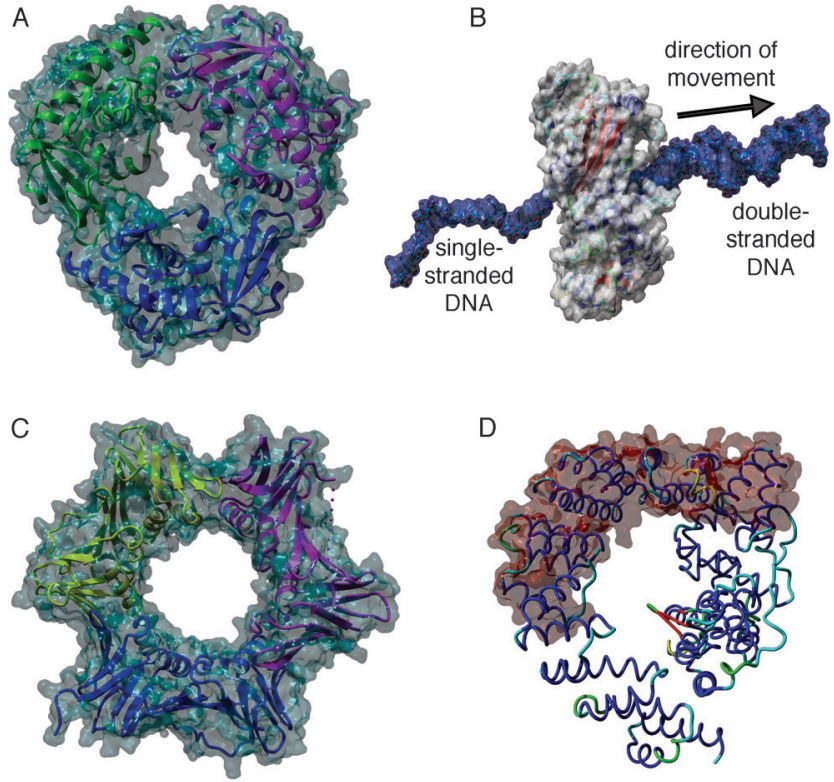

Figure 1. Substrate-enclosing proteins. A) Bacteriophage $\lambda$-exonuclease, ${ }^{[6]}$ a homotrimer. Note the small opening in its center back, which can only fit single-stranded DNA (PDB: 1AVQ). B) Impression of $\lambda$-exonuclease sliding over DNA, degrading only a single strand. C) S. solfataricus P2 PCNA, ${ }^{[9]}$ a sliding heterotrimer. Compare the larger central opening to that of $\lambda$-exonuclease. (PDB: 2IX2). D) Transglycosylase Slt70 from E. coli, ${ }^{[10]}$ an asymmetric toroid. The palm domain is highlighted in red, bottom left is the thumb, the fingers are on the right (PDB: 1QSA).

factor, archaically named proliferating cell nuclear antigen (PCNA; Figure 1c), is made up of subfactors 1, 2, and 3, which bind in a 1:1:1 stoichiometry. The complete heterotrimer, but not the individual subunits, binds the S. solfataricus DNA polymerase, its DNA ligase I, and its flap endonuclease. Thus a complete protein machine is recruited into a single processive architecture, where the processivity factor plays a key role as a tool belt and a guide. ${ }^{[9]}$

Next to symmetric toroids, some processive enzymes feature asymmetric rings. These rings are often compared with hands, featuring palm, thumb, and finger domains, as shown for the lytic transglycosylase Slt70 from E. coli (Figure 1d). Linked to the hand is the catalytic domain, which resembles a nonprocessive lysozyme that just happens to be linked to the ring. ${ }^{[10]}$ The hand allows the protein to encircle polysaccharide strands so that it can processively degrade cell wall polysaccharides until it encounters a crosslinked site. This site helps ensure that the chain ends in bacterial cell walls are crosslinked.

The T7 DNA polymerase also uses an asymmetric ring to bind to DNA, yet its finger domains cannot fully wrap around it. An additional processivity factor (thioredoxin) helps to stretch a finger, thus allowing full enclosure. ${ }^{[11]}$ Therefore, as described for $\mathrm{T} 4$ polymerase, closing of the ring also happens with $\mathrm{T} 7$ polymerase, where thioredoxin regulates processivity.

Regulation of polymerase processivity deserves some further comments. In general, clamp-shaped processivity factors are actively loaded onto a template by accessory clamp-loader proteins. ${ }^{[12]}$ They do not need to find the end of a nucleic acid chain to form a pseudorotaxane. These clamp loaders enable assembly of the entire replication complex wherever it is required, guided by recognition sequences. In DNA replication, polymerases only move across template strands in the $3^{\prime}$ to $5^{\prime}$ direction. The other strand is also replicated, but through multiple short Okazaki fragments (ca. 150 nucleotides in eukaryotes), which are later ligated. For this, many separate loading events are required, thus making processivity less vital. Studies have shown that some polymerases do indeed act distributively, ${ }^{[8,13]}$ depending on continuous recruitment and loading for ongoing polymerization. This behavior apparently suffices for the formation of short DNA fragments.

\subsection{Substrate-Binding Grooves}

Many processive enzymes only partially enclose their templates, thus making their structural basis for processivity less obvious. These non-topologically linked enzymes often possess a large groove or other type of binding domain for their substrate. Such a large surface offers advantages: the catalyst can undergo interactions with multiple adjacent sites on a single polymer, thus promoting specificity. Perhaps more importantly, the catalyst sliding along the template is promoted because association is based on multiple moderate interactions instead of on just a few strong ones.

Sliding dynamics are displayed by the chitinases grouped as family $18 .^{[14]}$ These enzymes have chitin-binding grooves (Figure 2a) and depolymerize their substrates, and is of great interest for biomass refinement. For insoluble substrates such
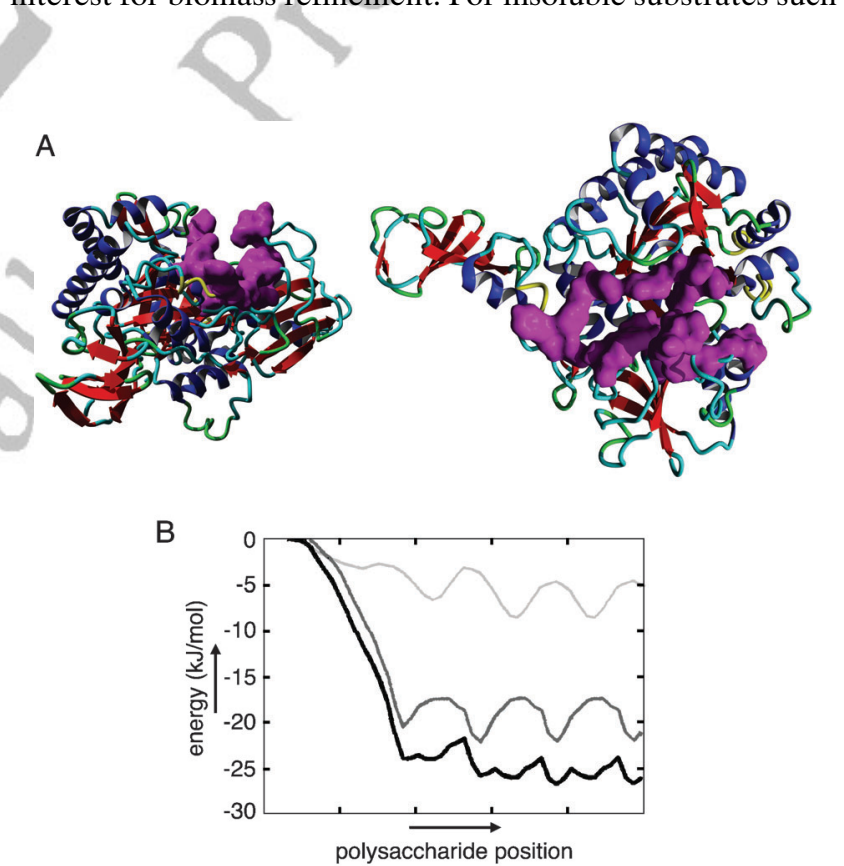

Figure 2. Chitinase and its substrate-binding groove..$\left.^{[14]} A\right)$ Two views of chitinase B from S. marcescens (PDB: 1E15). Residues involved in chitin binding are highlighted to reveal the groove. B) Energy profiles for a polysaccharide sliding through a protein. Aromatic stacking (light grey line) and hydrogen-bonding (dark grey line) combine in the total energy profile (black line), which is relatively smooth. Data originally published in the reference [15]. 
as chitin, processivity is essential because it helps prevent transiently dissolved individual chitin chains from re-associating with the bulk solid. It also precludes the need for reassociation after each turnover, a great boon for a substrate where binding is the highest energetic barrier. The fact that a groove is more accessible than a fully enclosed area putatively plays a role here.

Interactions between the enzyme and chitin are mostly hydrophobic, thus involving tyrosine residues in the groove and interact with both sides of the sugar ring, a common motif for carbohydrate-protein interaction. ${ }^{[14]}$ Hydrophobic interaction involves areas, and not individual binding partners as is the case for, for example, hydrogen bridges. This once more promotes sliding, though hydrogen bonds do play a prominent role in chitin recognition. Figure $2 b$ shows an interesting sliding energy profile, thereby detailing the contributions of $\pi-\pi$ stacking and of hydrogen bonding for a structurally related polysaccharide transporting pore ${ }^{[15]}$ Here $\pi-\pi$ stacking minima coincide with hydrogen-bonding maxima, thus resulting in a relatively smooth total energy profile. This interplay between different interactions probably allows chitin to slide more fluidly. ${ }^{[14]}$

Sliding is also important to the BamHI restriction endonuclease. It can recognize a specific DNA sequence, which it then hydrolyzes. ${ }^{[16]}$ BamHI displays higher catalytic activity rates than diffusion limits should allow, and is explained by its DNA-binding groove (Figure 3). BamHI
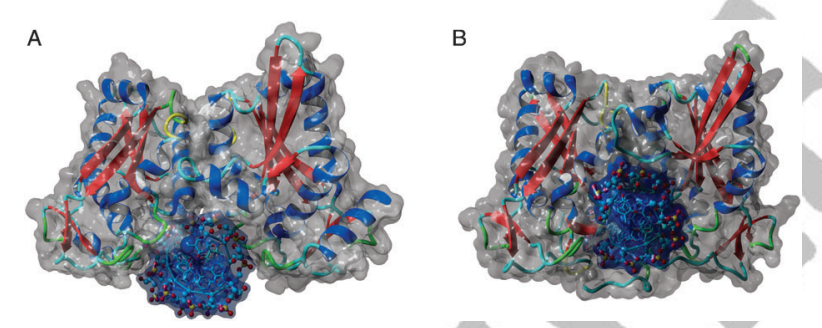

Figure 3. Two binding modes for Bam $\mathrm{HI} .^{[16]}$ A) Noncognate binding: BamHI straddles DNA (highlighted in blue) and slides along it (PDB 1ESG). B) At a recognition sequence, a conformational change locks the substrate DNA in place and opens up the active site (PDB: 3BAM).

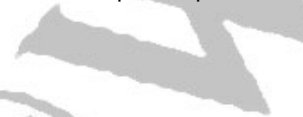

non specifically associates with DNA, straddling it like a saddle. It then slides along it until it recognizes its target sequence, which induces a conformational change: two $\alpha$ helices unwind, and wrap into the minor groove of the DNA, almost fully encircling it (Figure $3 \mathrm{~b}$ ). The noncognate binding mode, where BamHI slides in search of its target, involves only weak electrostatic interactions and no base pairs. Noncognate binding is sequence independent. The cognate binding mode is induced by the recognition sequence and involves protein-nucleotide interactions. It ultimately leads to scission. After hydrolysis, the enzyme can slide on to scan for further restriction sites. It is of interest that its processivity aids it not by supplying a conveyor belt of substrate, but by reducing three-dimensional diffusion limits into linear ones, thus making it more efficient. ${ }^{[17]}$ For certain systems, the exact contributions of intersubstrate distances and polymer tem- plate lengths have been investigated to fundamentally analyze the advantage of processivity. ${ }^{[18]}$ Thanks to its open groove, the sliding enzyme can hop over DNA-bound proteins, which would be impossible with full toroids. ${ }^{[19]}$

\subsection{Nonpolymeric Substrates}

Processivity is not limited to polymeric substrates. For example, pig pancreatic phospholipase $\mathrm{A}_{2}$ (PLA2) has a hydrophobic side for catalysis on a membrane interface. The active site opens on this side through a ring of cationic residues which help dissociate the anionic lipid substrates from the membranes. ${ }^{[20]}$ These membranes in turn shield PLA2's hydrophobic face from bulk solvent, thus making sure the enzyme remains associated with the membrane. In fact, when a suspension of lipid vesicles loaded with PLA2 was mixed with fresh vesicles, PLA2 was shown to not hop to the new vesicles. This behavior indicates that PLA2 is processive on a membrane substrate.

The vitamin-K-dependent carboxylase (VKDC) drives carboxylation of multiple glutamates in its substrate proteins to activate them as, for example, building blocks for protein matrices. ${ }^{[21]}$ Undercarboxylated substrates have been postulated to be detrimental to matrix formation, thus necessitating complete carboxylation of individual proteins. Fortunately, VKDC is processive, so premature dissociation of protein substrates from the enzyme seldom occurs. Its processivity is unlike any already discussed here: VKDC has an exosite that recognizes a specific protein sequence known as a propeptide (Figure 4). The propeptide binds tightly to the exosite, thus

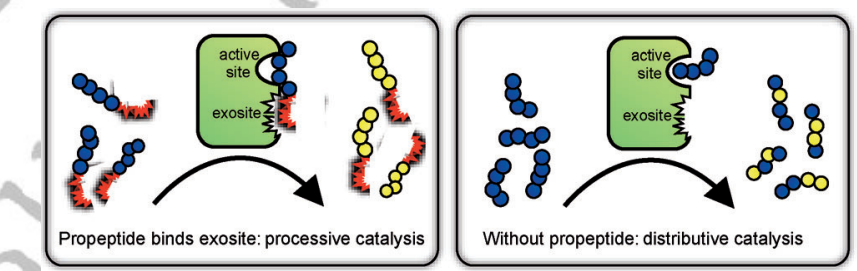

Figure 4. The basis of VKD carboxylase processivity. ${ }^{[2]]}$ A) A sequence in the substrate proteins (the propeptide) binds to an exosite in VKDC. The enzyme then converts all glutamate residues into the substrate. B) When substrates without a propeptide are offered, VKDC behaves distributively. The overall rate of catalysis increases, thus indicating that its processivity hinders turnover.

allowing the substrate protein ample residency time to offer all of its glutamates to the VKDC active site. When the propeptide dissociates, all glutamates in the substrate protein will have been carboxylated.

Studies where peptides with multiple glutamates, but no propeptide, were offered to VKDC showed that the turnover rate increased, but processivity was lost. This Doutcome implies that the propeptide actually slows down carboxylase activity because it might bind tighter than strictly necessary, thus needlessly blocking access to the active site when the currently bound substrate has already been converted. The 
processivity of VKDC thus appears to merely be based on a balance between binding affinity of its substrate and catalytic turnover speed. Yet, a more sophisticated mechanism might still play a role: carboxylation of the substrate might lead to increasing charge repulsion between it and the enzyme, thus causing a fully carboxylated substrate to dissociate much faster than a fresh one. Also, basic patches around the active site have been identified. Carboxylated sites in the substrate might bind to these patches and together trigger a conformational change in the VKDC exosite, thereby causing the propeptide to dissociate only when its protein has been comprehensively converted. ${ }^{[21]}$ The exact mechanism of processivity for VKDC will hopefully be revealed in the future, but the prospect of triggered release after processive conversion is exciting.

A final type of processivity is that of the cytochrome P450 family. It would be generous to describe cytochromes as truly processive, but for some pathways they certainly can be classified as such. For example, cytochrome P450 11B2 catalyzes the conversion of deoxycorticosterone into aldosterone in three steps without the substrate molecule dissociating in the meantime. ${ }^{[22]}$ However, other cytochrome enzymes can perform the same reactions, yet were shown to do so distributively, thereby making processivity in P450 cytochromes unpredictable. ${ }^{[23]}$ Chemists, then, might not be interested in drawing inspiration for artificial processive catalysts from $\mathrm{P} 450$ cytochromes. Nonetheless, their processivity is of relevance: medicinal chemist, who design drugs to target cytochrome pathways need to consider whether their target molecule is in fact a substrate, and not the intermediate of a processive catalytic sequence. Also, processive catalysis on small-molecule substrates is a rare phenomenon.

\subsection{Summary of Binding Modes in Nature}

Naturally processive enzymes which convert biopolymeric substrates either establish a mechanical link with them, or offer a large binding groove. ${ }^{[1]}$ Generally, substrate recognition appears to be nonspecific, which promotes catalyst sliding. Grooves readily form complexes, which is advantageous for ill-defined substrates (hydrophobic materials; ${ }^{[14]}$ foreign plasmids). ${ }^{[16]}$ Mechanical links offer higher processivity but require mediated complex formation, which is suitable for vital processes such as DNA replication. ${ }^{[7]}$

Non-biopolymeric substrates do not seem to follow general guidelines. They either appear as assembled membranes for enzymes to associate with, ${ }^{[20]}$ or contain dedicated recognition moieties which bind to exosites on the enzyme. ${ }^{[21]}$ They can even be so hydrophobic that they simply refuse to leave the active site until a certain amount of hydroxylation has taken place. ${ }^{[22]}$ Apparently, nature employs both method and fantasy when evolving processive enzymes.

\section{Artificial Processivity}

Attempts to emulate natural processivity are still in their infancy. Yet, a variety of achievements have been accom- plished, thus granting insight in various aspects of processivity.

\subsection{Macrocyclic Catalysts}

As reviewed in Ref. [2], the first catalytically active macrocyclic host to serve as a processive enzyme mimic was designed in the early 2000s. It combined a rigid, U-shaped receptor cavity with a diameter of about $9 \AA$ and a covalently linked manganese(III) porphyrin roof. The host was used to thread a polymeric alkene and processively epoxidize it (Figure $5 \mathrm{a}$ ). To ensure that catalysis only took place inside the

\section{A}
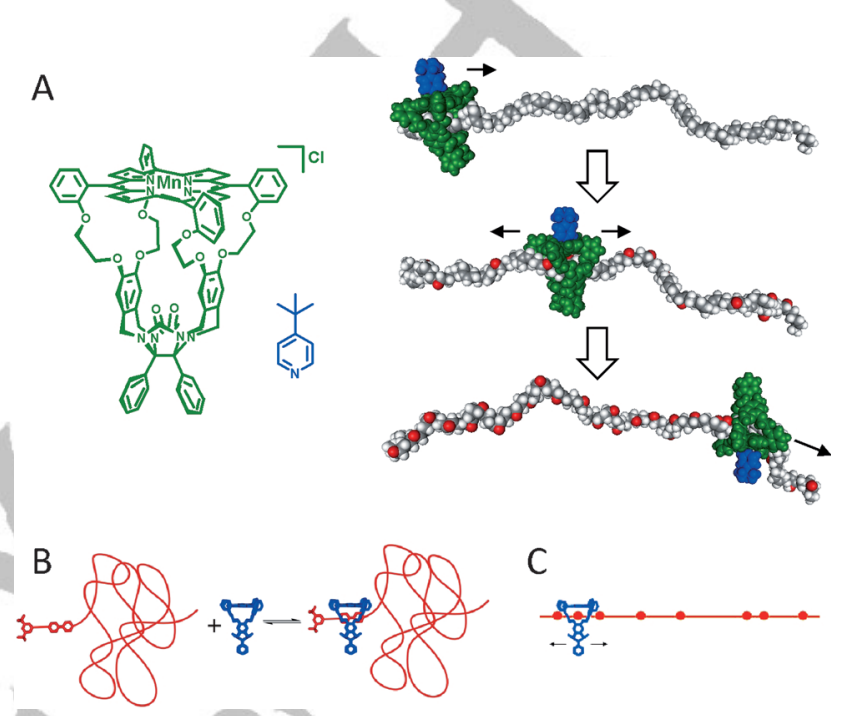

Figure 5. Processive epoxidation of polybutadiene by a catalytic macrocycle. ${ }^{[24]}$ A) Structure of the catalytic macrocycle (green) and the bulky axial ligand (blue; left). Sliding of the macrocycle/ligand complex from side to side over the polymer chain and epoxidation of the double bonds (right). B) Threading of a polymer that is blocked on one side; the macrocycle (blue) has to traverse the whole polymer chain to reach the viologen-binding station. C) Epoxidation of the polymer chain in a nonsequential processive fashion. See the cited review for references]..$^{[2}$

cavity, a bulky axial ligand (4-tert-butylpyridine), which coordinates to the manganese center but is too large to enter the cavity, was used. Axially coordinating substituents on the porphyrin also achieved this goal. ${ }^{[24]}$ In the presence of a single oxygen atom donor the catalyst fully converted polybutadiene $\left(M_{\mathrm{n}} 300000,98 \%\right.$ cis $)$ into its corresponding polyepoxide within 2 hours. This efficiency strongly suggested that the macrocycle performed its catalytic task in a pseudorotaxane-like topology, in which it slides over the polymer chain while carrying out epoxidation reactions in a processive fashion. To provide evidence for this mechanism, several control experiments were undertaken. Blocking of the cavity by $N, N^{\prime}$-dimethyl viologen, a guest with a high affinity for the receptor cavity, inhibited threading of the polymer and dramatically lowered the epoxidation rate. Second, the stereochemical outcome of a reaction catalyzed by the host ( $20 \%$ cis and $80 \%$ trans) was inverted when a reference manganese porphyrin catalyst, which lacked a receptor cavity, 
was used. This stereoselectivity was attributed to the confines of the cavity which apparently favors the sterically lessdemanding transition state and yields the trans epoxide as a result.

Being confident about the processive nature of the reaction, there was still the possibility that the polymer chain traversed the catalytic macrocycle by looping through the cavity and not by threading. In addition, the question whether the epoxidation of the polymer occurred in a sequential or a random fashion remained. To investigate these aspects, systematic threading studies were carried out using metal-free or zinc analogues of the macrocyclic host, with a series of welldefined chains which carried a viologen and a blocking group at one end. ${ }^{[25]}$ The only way for the macrocycle to reach the viologen end-of-the-line was by traversing the whole chain by threading over the open end (Figure $5 \mathrm{~b}$ ). This relatively slow process (in the order of minutes) could be monitored by NMR and fluorescence spectroscopy. These experiments provided unambiguous evidence for the threading mechanism, thus making the system the first synthetic processive catalytic (pseudo)rotaxane.

Another rotaxane-like catalyst was reported by the group of Harada. ${ }^{[2]}$ Cyclodextrins (CDs) were used as both a catalyst and as an artificial sliding clamp in the processive polymerization of $\delta$-valerolactone ( $\delta$-VL). $\beta$-CDs can include, activate, and open lactones, thus acting as a catalyst for $\delta$-VL polymerization. However, plain $\beta$-CDs only produce oligomers. To enable polymerization, the researchers linked a second $\mathrm{CD}$ to the $\beta$-CD active site, to help maintain contact between it and the nascent chain (Figure 6). This second CD,

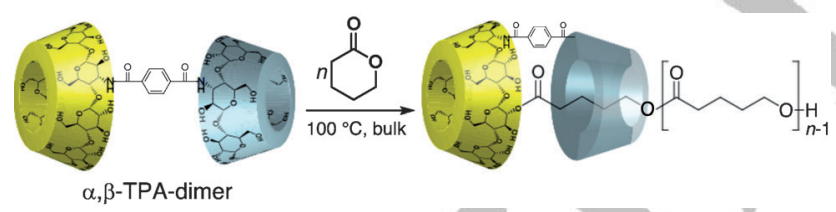

Figure 6. Processive polymerization of $\delta$-valerolactone enacted by a clamp/catalyst dimer. The clamp (steel blue, $\alpha$-cyclodextrin) is linked by a terephthalamide linker to the active site (yellow, $\beta-C D$ ). Reprinted with permission from Ref. [26].

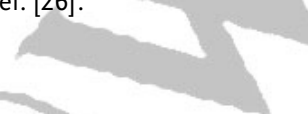

a smaller $\alpha-C D$, thus played the part of a sliding clamp by encircling the polymer chain and tethering the catalyst. This artificial molecular clamp reached conversions of up to $95 \%$, thus yielding polylactones with an $M_{\mathrm{n}}$ value of 16500 . The linker length was shown to be a relevant parameter in this process: short linkers had the tendency to suppress monomer recognition by the active-site $\mathrm{CD}$, while long linkers hindered the ability of the processivity-factor CD to clamp onto the nascent polymer chain. The optimal linker length was found to be about $7 \AA$, as provided by terephthalic acid.

The authors proved their proposed mechanism of action by adding adamantane as an inhibitor. Adamantane is included in $\beta$-CDs with high affinity, and indeed, polymerization was halted when the putative active site of the artificial clamp was blocked. More interestingly, the effect of the processivity factor (the noncatalytic $\alpha-C D$ ) was also gauged.
Because the nascent chain is linked to a hydroxy moiety of the $\beta-C D$, the clamp can be purified with a short oligomer already attached. Two-dimensional ROESY NMR experiments revealed that the nascent chain was indeed threaded through the $\alpha$-CD. When this complex was then used to re-initialize polymerization, conversions of $95 \%$ were once more achieved. However, when the oligomer was aun threaded from the $\alpha-C D$ by dissolving the complex in DMSO, subsequent attempts to re-initialize polymerization could not afford conversions higher than $3.5 \%$. The fact that the threaded state of the oligomer thus controls catalytic efficiency is an elegant proof of the actual processivity of this artificial clamp. ${ }^{[26]}$ It is reminiscent of how DNA polymerases can use both an enzyme and a separate dedicated processivity factor that fully encircles the substrate. ${ }^{[7]}$

A last ring-based system was reported in 2013 by the group of Leigh. ${ }^{[27]}$ Their rotaxane system comprised a macrocycle with a reactive arm and a template axle carrying a predetermined sequence of amino acids bound by weak phenolic ester linkages (Figure 7). The macrocycle was locked

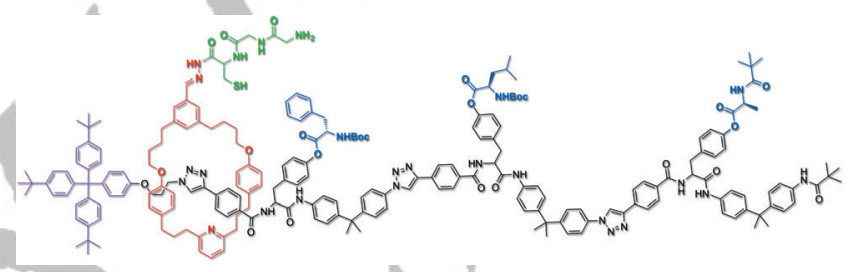

Figure 7. A processive rotaxane catalyst which clears a path for sliding by transferring labily linked amino acids to a nascent oligopeptide. ${ }^{[27]}$ Sliding macrocycle (red), active site (green), axle (black), stopper (magenta), and amino acid residues (blue).

in place by a large stopper group on one side, and the amino acids on the other. Through its reactive thiol arm it could pick up the axle amino acids and add them to a growing peptide, thus extending a peptide by up to three residues in a processive and sequential manner. Very recently, an improved synthesis of this functional rotaxane was reported, ${ }^{[28]}$ along with the ability to add a fourth residue. One of the main improvements was based on the fact that the original synthesis involved stoppering the macrocycle in place by conjugating the entire sequence-bearing template. This stoppering caused the challenging threading step to involve rather precious molecules. In the improved synthesis, the macrocycle was prethreaded on a shorter, easier to handle template where only the first amino acid was already locked in through its use as a stopper. The rest of the sequence was then added through extension in a follow-up step during which the macrocycle was already locked in place.

Because of its peptide synthesis, this machine distantly resembles a ribosome. However, its basis for processivity is more akin to that of the $\lambda$-exonuclease shown in Figure $1^{\left[{ }^{[6}\right.}$ Both are mechanically locked onto their templates and can only continue sliding if they first catalytically remove a residue which would otherwise block their paths. Thus, this smallmolecule machine successfully combines two of nature's strategies.

These are not the final page numbers! 


\subsection{Untethered Catalysts}

As described above, distributive enzymes can associate with processivity factors to become processive. Van Dongen and Clerx et al. applied this strategy to an artificial DNAnicking catalyst (a manganese tripyridyl porphyrin) by conjugating it to the $\mathrm{T} 4$ clamp protein discussed earlier (Figure 8). ${ }^{[2]}$ Apart from its new catalytic ability, the resulting

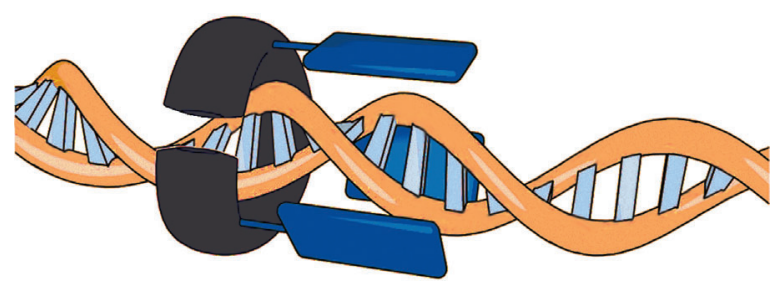

Figure 8. Cartoon representation of the T4 bacteriophage clamp protein (anthracite) noncognately bound to DNA. The clamp is trimeric, and each monomer is conjugated to a manganese tripyridyl porphyrin (blue) which nicks the DNA at AAA-sequence sites. The natural processivity of the clamp/DNA interaction is thus conferred on the otherwise distributive porphyrin catalyst. ${ }^{[29]}$

hybrid catalyst behaved just like the natural clamp: it could interact with clamp loader proteins which actively load the clamp on specific loader sites in DNA, and it could then slide over the DNA. The catalytic ability of the conjugated porphyrin, which intercalates and then nicks DNA at AAA sequences, combined with the clamp's sliding behavior to form a processive catalyst. Because of its noncognate sliding, during which the catalytic clamp searches for potential reaction sites, it strongly resembles the BamHI endonuclease. ${ }^{[16]}$ Instead of a protein conformational change to initiate a reaction, the tethered catalyst intercalates an AAA sequence, thus resulting in cognate binding. Nicking of the backbone releases the catalyst and allows further sliding.

In nature, the $\mathrm{T} 4$ clamp that was used recruits its polymerase through the polymerase $\mathrm{C}$ terminus by docking it into its open subunit interface (see Section 2.1). This docking stabilizes the clamp/DNA complex. When the researchers attempted to emulate this with a peptide analogue of the polymerase tail, the result was the opposite: while the peptide did close the open interface, it did so while the clamp was still in solution, thus resulting in a closed protein circle. This closed circle could no longer load onto DNA, thereby reverting the activity of the catalytic clamp to a distributive model. ${ }^{[2]}$ The desired stabilization of the clamp/ DNA complex remained out of reach, but the fact that the peptide allowed switching from a processive to a distributive mode of catalysis might be even more interesting.

Where the previous example added an artificial catalyst to an otherwise natural system, the next system is purely synthetic. Various polymerization reactions employ condensation catalysts, which might remain attached to the chains during polymerization. This aspect remained unexplored, until the group of Kiriy reported on the processivity of a nickel catalyst. ${ }^{[30]}$ It was used to polymerize 3-alkylthio-

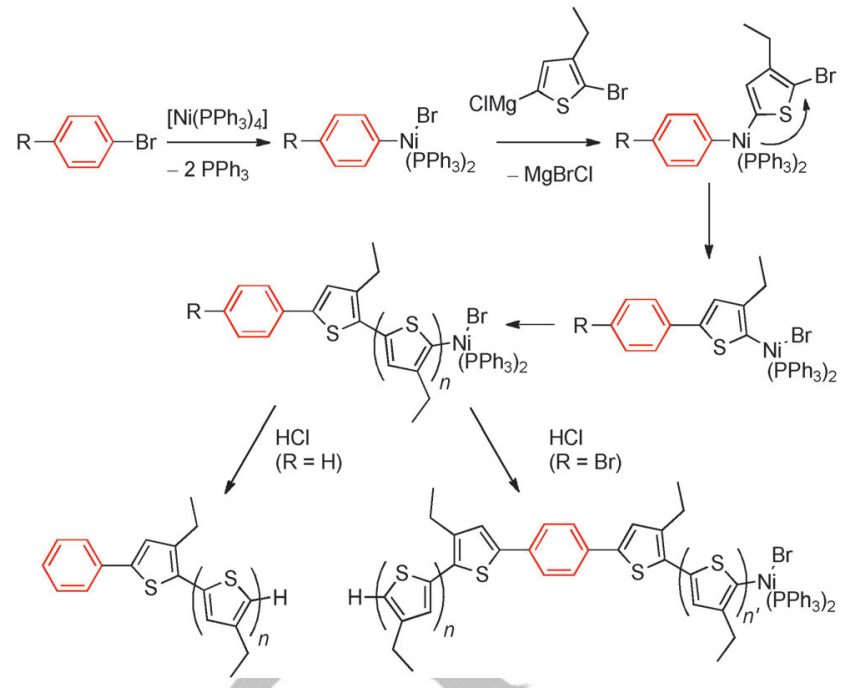

Scheme 1. A Kumada polycondensation is processively catalyzed by its nickel $(0)$ catalyst. After condensing thiophene monomers to one side of a 1,4-dibromobenzene initiator, the catalyst can walk over the polymer chain to start adding monomers to its other side as well. ${ }^{[31]}$

phene through a Kumada-type catalyst-transfer polycondensation. The mechanism of chain propagation involves transmetalation, reductive elimination, and oxidative addition (Scheme 1). The nickel catalyst may be called processive because in between catalytic cycles, it is eliminated but does not dissociate from the chain or react with other monomers. Instead, it undergoes oxidative addition into a $\mathrm{C}-\mathrm{Br}$ bond which is in the same chain. This processivity was first hinted at by the following experiment: polymer brushes were grown from surface-immobilized catalysts by dipping the surface in a monomer solution. Brush polymerization proceeded as designed, and no evidence of dissolved polymer was found. This $\square$ result $\square$ implies that all nickel catalysts remained surface-associated and did not leave their growing chains despite having the opportunity.

More definite proof, and an enjoyable demonstration of minimalistic catalyst sliding, was published later by Tkachov and co-workers. ${ }^{[31]}$ Inspired by the fact that the nickel catalyst has to walk over each monomer it just added to the growing chain, they used an initiator with not just a phenyl ring, but a $p$-bromophenyl ring which could grow a polymer from both its halides (Scheme 1). It was found that when the degree of polymerization $\left(D_{p}\right)$ was kept very low, this bromophenyl ring was found at chain ends, just as with the regular phenyl. With increasing $\mathrm{D}_{\mathrm{p}}$, the phenyl ring was found within the polymer chain, its prevalence increasing with the $\mathrm{D}_{\mathrm{p}}$. Polythiophene had grown from both halides. Since no intermolecular catalyst transfer takes place, these results show that the nickel catalyst slid along the polymer to the opposite end to initiate polymerization there. ${ }^{[31]}$

The group of Stojanovic provides us with a final example of an artificial processive catalyst, and it is again of a different type. ${ }^{[32]}$ The researchers assembled a star-shaped catalyst by attaching multiple DNA oligomers to a single protein center. Each attached oligomer was a short deoxyribozyme with the ability to hybridize with certain complimentary shorter 
chains. These recognition sites were then cleaved in two through the ribozymatic activity of the "leg". The four-legged catalyst could multivalently bind to a three-dimensional matrix covered with such substrates. It then diffused as follows: though each leg was bound to the matrix through substrate hybridization, its affinity would drop drastically after it cleaved its substrate, thus prompting its diffusion to an intact substrate. Thus, each leg could take individual steps, ensuring the catalyst would not dissociate from the matrix. In effect, it walked through it, cleaving substrates in its wake. This mechanism was demonstrated when a liquid flow was applied to the system: monovalent legs were shown to be flushed from the matrix rather rapidly, whereas multilegs remained present and active. While its mechanism is wholly different, this processive assembly resembles a phospholipase which diffuses over a membrane, cleaving its substrates as it goes. ${ }^{[20]}$

The researchers investigated the processive character further by studying the influence of leg length on dissociation. As expected, longer legs would result in more-tightly-bound catalysts, which, however, would have a lower turnover rate because new substrates would be less rapid in associating with the catalyst. As a result, the catalyst would diffuse more slowly, thus aggravating this effect. A second approach that increased the number of catalytic legs turned out to be more successful: the number of catalytic events generated by sixlegged molecules remained at the same high level, yet they were much less likely to be swept away in the flow. ${ }^{[32]}$ a nucleobase-linked vinyl monomer templated by complementary polymers, thus yielding daughter polymers with extremely low polydispersity $(\leq 1.08)$. While the only transferred information related to polymer length and not to the actual order of nucleobases, this different type of biomimicry is rather intriguing. Not only because increased monomer sequence control would allow the transfer of information, but also because it offers the potential to influence several macroscopic properties. Conductivity, elasticity, rigidity, or biodegradability are all dependent on monomer sequence,

a especially in water as a solvent. Please explain or remove. - For an in-depth discussion of sequential polymerization, we refer to a review by Lutz et al. ${ }^{[38]}$ While the full impact of efficient sequential catalysis cannot yet be fully anticipated, one thing appears to be certain: if templating and processivity were ever to meet, the results might be spectacular!

Processive catalysis, and processivity in general, is a vital principle in nature. Yet as we have seen, its chemical imitation or application are mostly still in the conceptual phase. If this field matures, processivity can become a choice when designing a catalyst, instead of a goal in itself. Polymer synthesis, post-modification, and degradation could be carried out in a far more efficient fashion, potentially revolutionizing many domains of chemistry.

Received: April 30, 2014

Published online: $\mathbf{\square}$ u, mu

\section{Outlook: Progress in Processivity}

While this review did not cover noncatalytic processivity, it might interest the reader to learn more about chemical imitations of walking motor proteins. Amongst others, the group of Seeman has used DNA origami technology to create a bipedal walker which could walk over a carefully organized DNA track. ${ }^{[33]}$ A more chemical approach to these walkers was reported by Von Delius et al., ${ }^{[34]}$ who made a 21 atom two-legged molecule which could walk up and down a fourstep molecular track. Each foot was either acid or base labile. This difference allowed them to be separately coaxed $\square$ coerced $\square$ into taking a step, that is, dissolving their original covalent link, and swaying towards the next available reactive position to bind there. Foot-track interactions were either based on hydrazone/aldehyde chemistry, or on disulfide bridge formation. A later iteration was based on metal-ion feet, where trans complexation drove the stepping, ${ }^{[35]}$ and a thermodynamic sink was shown to provide directionality to other random walkers. ${ }^{[36]}$

Another contemplation deals with sequentiality. Currently, the only artificial processive system with a proven sequence of catalytic events is the peptide synthesizer reported by Leigh and co-workers. ${ }^{[27]}$ In contrast, many natural processive systems are sequential-if not, DNA polymerization would not be able to replicate information. This a aspect $\mathbf{m a k e s}$ templated processes interesting to the field of processive catalysis. An example of such work hails from the group of O'Reilly, ${ }^{[37]}$ who reported the polymerization of
[1] W. A. Breyer, B. W. Matthews, Protein Sci. 2001, 10, 1699-1711.

[2] S. F. M. van Dongen, S. Cantekin, J. A. A. W. Elemans, A. E. Rowan, R. J. M. Nolte, Chem. Soc. Rev. 2013. $\square$ in press? $\square$

[3] A. Coskun, M. Banaszak, R. D. Astumian, J. F. Stoddart, B. A. Grzybowski, Chem. Soc. Rev. 2012, 41, 19-30.

[4] C. Veigel, C. F. Schmidt, Nat. Rev. Mol. Cell Biol. 2011, 12, 163 176.

[5] J. Frank, R. L. Gonzalez, Annu. Rev. Biochem. 2010, 79, 381 412.

[6] R. Kovall, B. W. Matthews, Science 1997, 277, 1824-1827.

[7] J. Yang, Z. Zhuang, R. M. Roccasecca, M. A. Trakselis, S. J. Benkovic, Proc. Natl. Acad. Sci. USA 2004, 101, 8289-8294.

[8] R. J. Bauer, I. D. Wolff, X. Zuo, H. K. Lin, M. A. Trakselis, J. Mol. Biol. 2013, 425, 4820-4836.

[9] I. Dionne, R. K. Nookala, S. P. Jackson, A. J. Doherty, S. D. Bell, Mol. Cell 2003, 11, 275-282.

[10] A. M. W. Thunnissen, H. J. Rozeboom, K. H. Kalk, B. W. Dijkstra, Biochemistry 1995, 34, 12729-12737.

[11] N. Q. Tran, S. J. Lee, B. Akabayov, D. E. Johnson, C. C. Richardson, J. Biol. Chem. 2012, 287, 39732-39741.

[12] A. Chakraborty, D. Wang, Y. W. Ebright, Y. Korlann, E. Kortkhonjia, T. Kim, S. Chowdhury, S. Wigneshweraraj, H. Irschik, R. Jansen, B. T. Nixon, J. Knight, S. Weiss, R. H. Ebright, Science 2012, 337, $591-595$.

[13] Z. Hu, S. K. Perumal, H. Yue, S. J. Benkovic, J. Biol. Chem. 2012, 287, 38442-38448.

[14] M. Sørlie, H. Zakariassen, A. L. Norberg, V. G. H. Eijsink, Biocatal. Biotransform. 2012, 30, 353-365.

[15] J. E. Meyer, G. E. Schulz, Protein Sci. 1997, 6, 1084-1091.

[16] H. Viadiu, A. K. Aggarwal, Mol. Cell 2000, 5, 889-895.

[17] N. Shimamoto, J. Biol. Chem. 1999, 274, 15293-15296.

[18] M. A. Surby, N. O. Reich, Biochemistry 1996, 35, 2201-2208.

[19] M. Hedglin, P. J. O’Brien, ACS Chem. Biol. 2010, 5, 427-436. 
[20] F. Ramirez, M. K. Jain, Proteins Struct. Funct. Bioinf. 1991, 9 , 229-239.

[21] M. A. Rishavy, K. L. Berkner, Adv. Nutr. 2012, 3, 135-148.

[22] T. Imai, T. Yamazaki, S. Kominami, Biochemistry 1998, 37, 8097 8104.

[23] F. P. Guengerich, C. D. Sohl, G. Chowdhury, Arch. Biochem. Biophys. 2011, 507, 126-134.

[24] C. Monnereau, P. H. Ramos, A. B. C. Deutman, J. A. A. W. Elemans, R. J. M. Nolte, A. E. Rowan, J. Am. Chem. Soc. 2010, 132, 1529-1531.

[25] A. B. C. Deutman, C. Monnereau, J. A. A. W. Elemans, G. Ercolani, R. J. M. Nolte, A. E. Rowan, Science 2008, 322 , $1668-1671$.

[26] Y. Takashima, M. Osaki, Y. Ishimaru, H. Yamaguchi, A. Harada, Angew. Chem. 2011, 123, 7666-7670; Angew. Chem. Int. Ed. 2011, 50, 7524-7528.

[27] B. Lewandowski, G. De Bo, J. W. Ward, M. Papmeyer, S. Kuschel, M. J. Aldegunde, P. M. E. Gramlich, D. Heckmann, S. M. Goldup, D. M. D'Souza, A. E. Fernandes, D. A. Leigh, Science 2013, 339, 189-193.

[28] G. De Bo, S. Kuschel, D. A. Leigh, B. Lewandowski, M. Papmeyer, J. W. Ward, J. Am. Chem. Soc. 2014, 136, 5811-5814.
[29] S. F. M. van Dongen, J. Clerx, K. Nørgaard, T. G. Bloemberg, J. J. L. Cornelissen, M. A. Trakselis, S. W. Nelson, S. J. Benkovic, A. E. Rowan, R. J. M. Nolte, Nat. Chem. 2013, 5, 945-951.

[30] V. Senkovskyy, N. Khanduyeva, H. Komber, U. Oertel, M. Stamm, D. Kuckling, A. Kiriy, J. Am. Chem. Soc. 2007, 129, $6626-6632$.

[31] R. Tkachov, V. Senkovskyy, H. Komber, J. U. Sommer, A. Kiriy, J. Am. Chem. Soc. 2010, 132, 7803-7810.

[32] R. Pei, S. K. Taylor, D. Stefanovic, S. Rudchenko, T. E. Mitchell, M. N. Stojanovic, J. Am. Chem. Soc. 2006, 128, 12693-12699.

[33] T. Omabegho, R. Sha, N. C. Seeman, Science 2009, 324, 67-71.

[34] M. von Delius, E. M. Geertsema, D. A. Leigh, Nat. Chem. 2010, 2, 96-101.

[35] J. E. Beves, V. Blanco, B. A. Blight, R. Carrillo, D. M. D'Souza, D. Howgego, D. A. Leigh, A. M. Z. Slawin, M. D. Symes, J. Am. Chem. Soc. 2014, 136, 2094-2100.

[36] A. G. Campaña, D. A. Leigh, U. Lewandowska, J. Am. Chem. Soc. 2013, 135, 8639-8645.

[37] R. McHale, J. P. Patterson, P. B. Zetterlund, R. K. O'Reilly, Nat. Chem. 2012, 4, 491-497.

[38] J. F. Lutz, M. Ouchi, D. R. Liu, M. Sawamoto, Science 2013, 341, 1238149 


\section{Minireviews}

Enzyme Catalysis

S. F. M. van Dongen,*

J. A. A. W. Elemans, A. E. Rowan,

R. J. M. Nolte*

III-IIII

Processive Catalysis

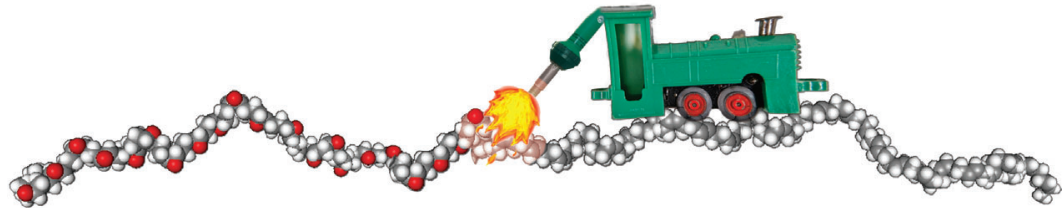

Hold the line: In processive catalysis, a catalyst binds to its substrate and performs multiple rounds of catalysis before dissociation. Nature leverages this phenomenon in its synthesis or processing of biopolymers. Processivity allows achievement of rates of catalysis which cannot be matched by distributive systems. This review describes processive catalysis and the advances that have been made in emulating it through supramolecular chemistry. 